\title{
Proposed Neurobiological Processes Associated with Models of Vertebral Subluxation: Dysafferentation, Dyskinesia, Dysponesis, Dysautonomia, Neuroplasticity and Ephaptic Transmission
}

\author{
Christopher Kent* \\ Director of Evidence-Informed Curriculum and Practice, USA
}

*Corresponding author: Christopher Kent, Director of Evidence-Informed

Curriculum and Practice, Sherman College of Chiropractic, Spartanburg, South Carolina, USA.

Received Date: March 25, 2019

Published Date: April 04, 2019

\begin{abstract}
Vertebral subluxations are changes in the position or motion of a vertebra, which result in the interference with nerve function. This paper describes possible neurobiological mechanism associated with vertebral subluxations. These mechanisms include dysafferentation, dyskinesia, dysponesis, dysautonomia, neuroplasticity and ephaptic transmission.
\end{abstract}

Keywords: Vertebral subluxation; Dysafferentation; Dyskinesia; Dysponesis; Dysautonomia; Neuroplasticity; Ephaptic transmission; Chiropractic

\section{Introduction}

Vertebral subluxations are changes in the position or motion of a vertebra, which result in the interference with nerve function [1]. As Lantz [2] noted, "Common to all concepts of subluxation are some form of kinesiologic dysfunction and some form of neurologic involvement." According to Stephenson's 1927 definition [3], vertebral subluxation is: "the condition of a vertebra that has lost its proper juxtaposition with the one above the one below, or both; to an extent less than a luxation; which impinges nerves and interferes with the transmission of mental impulses."

In clinical practice, conceptual definitions, such as those described above, must give rise to operational definitions. An operational definition is a description of the procedures used to determine the means for measuring or observing something [4]. Smith et al. stated, "The potential exists for subluxation resolution to be conceptualized as a legitimate intermediate health outcome, pending the development of a sufficient and requisite body of scientifically derived clinical evidence [5]. This body of evidence must, by necessity, include [1] scientifically valid and reliable measures of subluxation, in order to [2] scientifically examine the relationship between a patient's subluxation and that patient's health".

The author has proposed an operational model for the assessment of neurological dysregulation associated with vertebral subluxation [6]. The four components of this model are dysafferentation, dyskinesia, dysponesis, and dysautonomia. In the context of dysafferentation, neuroplasticity may be considered. In addition to these mechanisms, non-synaptic communications are potentially affected by vertebral subluxations.

\section{Discussion}

Physical mechanisms associated with signaling in the human body

Hewitt [7] proposed a classification of physical mechanisms associated with signaling in the human body:

1. Diffusion of particles along concentration gradients. This would include processes associated with membrane potentials and ionic exchange. 
2. Diffusion of quanta along electromagnetic gradients. These processes are associated with electrical potentials and fields, and include EEG, ECG, EMG, and field effects of ephaptic transmission.

3. Circulation within structured channels. Examples include blood, lymph, cerebrospinal fluid [CSF] and axoplasmic flow.

4. Wave propagation. Longitudinal and transverse waves, as seen in vibratory sense, audition, and soliton waves.

These physical mechanisms are operative in both linear, synaptic processes and nonlinear, nonsynaptic processes.

\section{Dysafferentation}

The intervertebral motion segment is richly endowed with nociceptive and mechanoreceptive structures [8-13]. As a consequence, biomechanical dysfunction caused by vertebral subluxation may result in altered nociception and/ or mechanoreception. Aberrant afferent input to the CNS may result in qualitatively and/or quantitatively inappropriate responses to changes in the internal or external environment. This is known as dysafferentation [14].

Himes and Peterson [15] proposed the concept of a "neural image," dependent upon the integrity of neural receptors and afferent pathways. This "neural image" is a representation of the organism's perception of the external and internal environment. If afferent input is compromised, efferent response may be qualitatively and quantitatively compromised.

\section{Neuroplasticity}

The clinical implications of aberrant or suboptimal afferent go beyond short-term homeostatic regulation. Dysafferentation may result in anatomical and functional changes in the brain itself. Merzenich, quoted in Holloway stated, [16] "The brain was constructed to change." This challenge to the conventional world view that the mature adult brain is stable and unchanging, the only exception being the death of brain cells, has profound clinical implications.

Furthermore, Holloway explained how the brain reconfigures itself and the implications of doing so: "Change the input, be it a behavior, a mental exercise ... or a physical skill and the brain changes accordingly. Magnetic resonance imaging machines reveal the new map: different regions light up ... The brain can be extensively remodeled throughout the course of one's life, without drugs, without surgery. Regions of the brain can be taught to do different tasks if need be ... This sort of thing will be a part of normal future life ... healing plasticity can be driven by behavior."

Gage [17] wrote, "Researchers first demonstrated that the central nervous systems of mammals contain some innate regenerative properties in the 1960s and 1970s, when several groups showed that axons, or main branches, of neurons in the adult brain and spinal cord can regrow to some extent after injury." The ability of the brain to change both anatomically and functionally is known as neuroplasticity. Clifford [18] described three types of plasticity:

- Experience-independent plasticity refers to changes which are not the result of environmental changes or influence.

- Experience-expectant plasticity occurs when the brain uses input from the external environment to effect normal developmental changes in its structure.

- $\quad$ Experience-dependent plasticity is when a modification to the internal or external environment produces change in a feature of the brain.

However, not all neuroplastic changes are favorable. It has been hypothesized that maladaptive neuroplastic changes may be associated with vertebral subluxations [19].

\section{Dyskinesia}

Dyskinesia refers to distortion or impairment of voluntary movement [20]. Spinal motion may be reliably measured using inclinometry [21]. Alterations in regional ranges of motion may be associated with vertebral subluxation [22].

\section{Dysponesis}

Dysponesis is evidenced by abnormal tonic muscle activity. Dysponesis refers to a reversible physiopathologic state consisting of errors in energy expenditure, which is capable of producing functional disorders. Dysponesis consists mainly of covert errors in action potential output from the motor and premotor areas of the cortex and the consequences of that output. These neurophysiological reactions may result from responses to environmental events, bodily sensations, and emotions. The resulting aberrant muscle activity may be evaluated using surface electrode techniques [23]. Typically, static SMEG with axial loading is used to evaluate innate responses to gravitational stress [24]. Surface EMG procedures are reliable [25].

\section{Dysautonomia}

The autonomic nervous system regulates the actions of organs, glands, and blood vessels. Acquired dysautonomia may be associated with a broad array of functional abnormalities [26-31]. Sympathetic tone may be evaluated by measuring skin temperature differentials using paraspinal infrared thermography [32]. Such techniques have been used to monitor changes in neurological function associated with vertebral subluxations [33] and are reliable [34]. Heart rate variability is a reliable and valid technique for the assessment of changes in autonomic nervous system function $[35,36]$.

\section{Ephaptic Transmission}

Ephaptic transmission occurs when electric fields activate neighboring cells without synaptic transmissions or gap junctions [37]. Boone and Dobson [38] discussed mechanisms of non-synaptic transmission, including ephaptic coupling, as a mechanism which may be affected by vertebral subluxation. However, technology to assess this phenomenon clinically is lacking, and the role of 
ephaptic transmission in cases of vertebral subluxation remains speculative.

\section{Conclusion}

Correction or reduction of vertebral subluxation facilitates the restoration of proper tone throughout the nervous system. Alterations in the tone of the somatic system may be objectively evaluated using surface EMG. Altered autonomic tone may be evaluated using skin temperature measurements. Changes in ranges of motion may be measured to assess dyskinesia. Such objective assessments have the potential to make correction of vertebral subluxation an important strategy in salutogenic healthcare. Additional research in this area may lead to improved clinical strategies.

\section{Acknowledgment}

\section{None}

\section{Conflict of Interest}

No conflict of interest.

\section{References}

1. Kent C (2018) Chiropractic and mental health: History and review of putative neurobiological mechanisms. Journal of Neurology, Psychiatry and Brain Research JNPB-103: 1-10.

2. Lantz CA (1995) The subluxation complex. In: Gatterman MI (Ed.), Foundations of Chiropractic Subluxation. St. Louis, MO: Mosby, USA

3. Stephenson RW (1927) Chiropractic Text-Book. Davenport, IA: Palmer School of Chiropractic, USA.

4. Meeker WC (2000) Concepts germane to evidence-based application of chiropractic theory. Top Clin Chiropr 7: 67-73.

5. Smith M and Gromola TJ (2001) The role of subluxation in delivering quality chiropractic health care. Top Clin Chiropr 8: 29-33.

6. Kent C (2017) Heart rate variability to assess the changes in autonomic nervous system function associated With vertebral subluxation. Research \& Reviews: Neuroscience 1(3): 14-21.

7. Hewitt WF (1969) Somatic aspects of applied physiology. In: Hoag JG (Ed.), Osteopathic Medicine. McGraw Hill Book Company. New York, USA.

8. Bogduk N, Tynan W, Wilson AS (1981) The nerve supply to the human lumbar intervertebral discs. J Anat 132: 39-56.

9. Nakamura S, Takahashi K, Takahashi Y, Shimada Y, Moriya H (1996) Origin of nerves supplying the posterior portion of lumbar intervertebral discs. Spine 21(8): 917-924.

10. Mendel T, Wink CS, Zimny ML (1992) Neural elements in human cervical intervertebral discs. Spine 17: 132-135.

11. McLain RF (1994) Mechanoreceptor endings in human cervical facet joints. Spine 19: 495-501.

12. Jiang H, Russell G, Raso VJ, Moreau MJ, Hill DL, et al. (1995) The nature and distribution of the innervation of human supraspinal and interspinal ligaments. Spine 20: 869-876.

13. Rhalmi S, Yahia LH, Newman N, Isler M (1993) Immunohistochemica study of nerves in lumbar spine ligaments. Spine 18: 264-267.

14. Kent C (1996) Models of vertebral subluxation: a review. Journal of Vertebral Subluxation Research. August 1: 1-7.

15. Segmental Neuropathy (no date) Canadian Memorial Chiropractic College. Toronto, Ontario.
16. Holloway M (2002) The mutable brain. Sci Am 289(3): 79.

17. Gage FH (2002) Brain, repair yourself. Sci Am 289(3): 47.

18. Clifford E, Merzenich, Taub, Greenough (2009) The Harvard Brain Neural Plasticity 6: 16-20.

19. Holt K, Niazi IK, Nedergaard RW, Duehr J, Muhammad S, et al. (2019) The effects of a single session of chiropractic care on strength, cortical drive, and spinal excitability in stroke patients. Sci Rep 9(1): 2673.

20. Dorland's Pocket Medical Dictionary (1995) $25^{\text {th }}$ edition. WB Saunders Company. Philadelphia, PA, USA.

21. Saur PM, Ensink FB, Frese K, Seeger D, Hildebrandt J (1996) Lumbar range of motion: reliability and validity of the inclinometer technique in the clinical measurement of trunk flexibility. Spine 21: 1332-1338.

22. Blunt KL, Gatterman MI, Bereznick DE (1995) Kinesiology: an essential approach toward understanding the chiropractic subluxation. Chapter 11. In: Gatterman MI (Ed.), Foundations of Chiropractic Subluxation. Mosby, St. Louis, MO, USA

23. Whatmore GB, Kohi DR (1968) Dysponesis: a neurophysiologic factor in functional disorders. Behav Sci 13: 102-124.

24. Large R, Butler M, James F, Peters J (1990) A systems model of chronic musculo-skeletal pain. Aust N Z J Psychiatry 24(4): 529-536.

25. Kent C (1997) Surface electromyography in the assessment of changes in paraspinal muscle activity associated with vertebral subluxation: a review. Journal of Vertebral Subluxation Resarch 1:15.

26. Backonja M-M (1994) Reflex sympathetic dystrophy/sympathetically mediated pain/causalgia: the syndrome of neuropathic pain with dysautonomia. Seminars in Neurology 14: 263-271.

27. Goldstein DS, Holmes C, Cannon III RO, Eisenhofer G, Kopin IJ (1997) Sympathetic cardioneuropathy in dysautonomias. New Engl J Med 336: 696-702.

28. Vassallo M, Camilleri M, Caron BL, Low PA (1991) Gastrointestinal motor dysfunction in acquired selective cholinergic dysautonomia associated with infectious mononucleosis. Gastroenterology 100: 252-258.

29. Baron R, Engler F (1996) Postganglionic cholinergic dysautonomia with incomplete recovery: a clinical, neurophysiological and immunological case study. J Neurol 243: 18-24

30. Soares JLD (1996) Dysautonomias. Acta Medica Portuguesa 8: 425.

31. Stryes KS (1994) The phenomenon of dysautonomia and mitral valve prolapse. J Am Acad Nurse Practitioners 6: 11-15.

32. Uematsu S, Edwin DH, Jankel ER, Kozikowski J, Trattner M (1988) Quantification of thermal asymmetry. J Neurosurg 69(4): 552-555.

33. Miller JL (1964) Skin temperature differential analysis. International Review of Chiropractic. Science 1: 41.

34. McCoy M, Campbell I, Stone P, Fedorchuk C, Wijayawardana S, et al (2011) Intra-examiner and inter-examiner reproducibility of paraspinal thermography. PLoS One 6(2): e16535.

35. Nakamura FY, Pereira LA, Esco MR, Flatt AA, et al. (2017) Intraday and Interday Reliability of Ultra-Short-Term Heart Rate Variability in Rugby Union Players. J Strength Cond Res 31(2): 548-551.

36. Williams DP, Jarczok MN, Ellis RJ, Hillecke TK, et al (2017) Two-week test-retest reliability of the Polar ${ }^{\circledR}$ RS800CX ${ }^{\mathrm{TM}}$ to record heart rate variability. Clin Physiol Funct Imaging 37(6): 776-781.

37. Chiang CC, Shivacharan RS, Wei X, Gonzalez-Reyes LE, Durand DM (2019) Slow periodic activity in the longitudinal hippocampal slice can self-propagate non-synaptically by a mechanism consistent with ephaptic coupling. J Physiol 597(1): 249-269.

38. Boone WR Dobson GJ (1996) A proposed vertebral subluxation mode reflecting traditional concepts and recent advances in health and science. Journal of Vertebral Subluxation Research 1(1): 1-12. 